\title{
Square-free words as products of commutators
}

\author{
Andrew Duncan and Alina Vdovina
}

\begin{abstract}
Elements of the commutator subgroup of a free group $\mathbb{F}$ can be presented as values of canonical forms, called Wicks forms. We show that, starting from sufficiently high genus $g$, there is a sequence of words $w_{g}$ which can be presented by $f(g)$ distinct Wicks forms, where $f(g)>g$ !. Moreover we may choose these words $w_{g}$ to be square-free.
\end{abstract}

Mathematics Subject Classification (2000). 20E05, 20F10.

Keywords. Commutators, free group, square-free words.

\section{Introduction}

Let $\mathbb{F}$ be a free group and let $[\mathbb{F}, \mathbb{F}]$ be its commutator subgroup. We define the genus of a word $w \in[\mathbb{F}, \mathbb{F}]$ to be the least positive integer $g$ such that $w$ is a product of $g$ commutators in $\mathbb{F}$. Every element of genus $g$ in $[\mathbb{F}, \mathbb{F}]$ can be presented by noncancelling substitution in a Wicks form of genus $g$ ([cf. [4], [3]]) as described below. In genus one there is only one Wicks form but for $g \geq 2$ there are finitely many Wicks forms and their number grows quite fast (factorially) with $g$. Thus a natural question (posed by E. Rips at the geometric group theory conference in Anogia in 1996) is whether it is possible to find a word of genus $g$ which can be presented by non-cancelling substitution in "many" Wicks forms. We show that there is a sequence of words $w_{2}, w_{3}, \ldots$ such that $w_{g}$ has genus $g$ and the number of Wicks forms from which it can be obtained by non-cancelling substitution is bounded below by $g$ !, when $g$ is sufficiently large.

Wicks forms are not affected by Dehn twists. Since, by a classical result of M. Dehn ([5]), the modular group is generated by Dehn twists, Wicks forms are invariant under the action of $\operatorname{Mod}\left(S_{g}\right)$. In [2] Bestvina and Feighn consider genus $g$ representations (defined below) of words of genus $g$. They describe an equivalence relation on representations in terms of partial Dehn twists, which are a generalisation of Dehn twists. They show that for certain types of word $w_{g}$ the number of distinct equivalence classes of representations of $w_{g}$ grows exponentially with $g$. It would be interesting to know what happens to our Wicks form representations under fractional Dehn twists. 


\section{Wicks forms}

An alphabet consists of a countably enumerable set $S$ equipped with a fixed-point free involution $\tau$ and a fixed set of representatives $S^{+}$of the orbits of $\tau$. We shall consider words in a fixed alphabet $A=\left\{a_{1}^{ \pm 1}, a_{2}^{ \pm 1}, \ldots\right\}$ of letters $a_{1}, a_{2}, \ldots$ and their inverses $a_{1}^{-1}, a_{2}^{-1}, \ldots$, where $\tau\left(a_{i}\right)=a_{i}^{-1}$ and $\tau\left(a_{i}^{-1}\right)=a_{i}$ and $A^{+}=\left\{a_{1}, a_{2}, \ldots\right\}$. Alphabets $B, A^{\prime}$ are defined analogously, replacing $a_{i}$ by $b_{i}$ or $a_{i}^{\prime}$, in the obvious way. A word (over $A$ ) is an element of the free monoid $A^{*}$ on $A$. A cyclic word (over $A$ ) is the set of cyclic permutations $[w]$ of a word $w$. Words $w$ and $w^{\prime}$ are said to define the same cyclic word if $[w]=\left[w^{\prime}\right]$. A word $u$ is said to be a factor of the cyclic word [w] if $u$ is a subword of some element of [w]. A word is said to be reduced if it has no factor of the form $a a^{-1}$ or $a^{-1} a$, where $a \in A^{+}$. A word is said to be cyclically reduced if every element of $[w]$ is reduced. An element $a^{\epsilon}$, where $a \in A^{+}$ and $\epsilon= \pm 1$, is said to occur n-times in the word $w$ if $w=u_{1} a^{\epsilon} u_{2} \ldots u_{n} a^{\epsilon} u_{n+1}$, for some elements $u_{1}, \ldots, u_{n+1}$ of $\left(A \backslash\left\{a^{\epsilon}\right\}\right)^{*}$ : we count the number of occurrences of $a^{+1}$ and $a^{-1}$ separately.

Definition 1. An orientable Wicks form is a cyclic word [ $w]$ over $A$ such that

(i) if $a^{\epsilon} \in A$ occurs in $w$ (for $a \in A^{+}$and $\epsilon \in\{ \pm 1\}$ ) then $a^{-\epsilon}$ occurs exactly once in $w$;

(ii) $w$ is cyclically reduced, and

(iii) if $a_{i}^{\epsilon} a_{j}^{\delta}$ is a factor of $[w]$ then $a_{j}^{-\delta} a_{i}^{-\epsilon}$ is not a factor of $[w]$.

We shall abuse notation by referring to a Wicks form $[w]$ as $w$ when convenient. An orientable Wicks form $[w]=\left[w_{1} w_{2} \ldots\right]$, over $A$, is isomorphic to $\left[w^{\prime}\right]=\left[w_{1}^{\prime} w_{2}^{\prime} \ldots\right]$, over the alphabet $A^{\prime}$, if there exists a bijection $\varphi: A \rightarrow A^{\prime}$ with $\varphi\left(a^{-1}\right)=\varphi(a)^{-1}$ such that $[\varphi(w)]=\left[w^{\prime}\right]\left(\right.$ where $\left.\varphi(w)=\varphi\left(w_{1}\right) \varphi\left(w_{2}\right) \ldots\right)$. The relation "is isomorphic to" is an equivalence relation on the orientable Wicks forms over $A$.

If $[w]$ is an orientable Wicks form then $w$, when considered as an element of the free group $\mathbb{F}$ generated by $a_{1}, a_{2}, \ldots$, is an element of the commutator subgroup. We define the algebraic genus $g_{a}(w)$ of $[w]$ to be the least positive integer $g_{a}$ such that $w$ is a product of $g_{a}$ commutators in $\mathbb{F}$.

The topological genus $g_{t}(w)$ of an orientable Wicks form $w=w_{1} \ldots w_{2 e-1} w_{2 e}$ is defined to be the topological genus of the orientable compact connected surface obtained by labelling and orienting the edges of a $2 e-$ gon (which we consider as a subset of the oriented plane) according to $w$ and then identifying edges with the same labels (respecting orientation).

Proposition (cf. [4], [3]). The algebraic genus and the topological genus of an orientable Wicks form coincide.

We define the $\operatorname{genus} g(w)$ of an orientable Wicks form $[w]$ to be $g(w)=g_{a}(w)=$ $g_{t}(w)$. 
Consider the orientable compact surface $S$ associated to an orientable Wicks form $w=w_{1} \ldots w_{2 e}$. This surface carries an embedded graph $\Gamma \subset S$ such that $S \backslash \Gamma$ is an open polygon with $2 e$ sides (and hence connected and simply connected). Moreover, conditions (ii) and (iii) on the Wicks form imply that $\Gamma$ contains no vertices of degree 1 or 2 (or equivalently that the dual graph of $\Gamma \subset S$ contains no faces which are 1-gons or 2-gons). This construction also works in the opposite direction: given a graph $\Gamma \subset S$ with $e$ edges and no vertices of degree 1 or 2 on an orientable compact connected surface $S$ of genus $g$ such that $S \backslash \Gamma$ is connected and simply connected, we get an orientable Wicks form of genus $g$ and length $2 e$ by labelling and orienting the edges of $\Gamma$ and by cutting $S$ open along the graph $\Gamma$. The associated orientable Wicks form is defined as the word which appears in this way on the boundary of the resulting polygon with $2 e$ sides. Henceforth we identify orientable Wicks forms with their associated embedded graphs $\Gamma \subset S$, speaking of vertices and edges of orientable Wicks forms.

The formula for the Euler characteristic

$$
\chi(S)=2-2 g=v-e+1
$$

(where $v$ denotes the number of vertices and $e$ the number of edges in $\Gamma \subset S$ ) shows that an orientable Wicks form of genus $g$ has length at least $4 g$ (the associated graph has then a unique vertex of degree $4 g$ and $2 g$ edges) and length at most $6(2 g-1)$ (the associated graph has then $2(2 g-1)$ vertices of degree three and $3(2 g-1)$ edges).

We call an orientable Wicks form of genus $g$ maximal if it has length $6(2 g-1)$. It is convenient to interpret a genus $g$ Wicks form as an oriented circuit in the graph $\Gamma$; that is a circuit which traverses every edge of the graph exactly twice, once in each direction, but contains no instance of an edge followed by its reverse (for more details concerning oriented circuits see [9]). The dual graph of a Wicks form is an ideal triangulation, as defined by L. Mosher [6]. It is shown in [6] that ideal triangulations are invariant under Dehn twists.

Let $g$ be a positive integer, $A=\left\{a_{1}^{ \pm 1}, \ldots, a_{6 g-3}^{ \pm 1}\right\}$ and $B=\left\{b_{1}^{ \pm 1}, \ldots, b_{n}^{ \pm 1}\right\}$ be alphabets and let $A^{*}$ and $B^{*}$ be the free monoids on $A$ and $B$, respectively. Define $\left(a_{i}^{-1}\right)^{-1}=a_{i}$ and for a word $w=x_{1} \ldots x_{n} \in A^{*}$ with $x_{i} \in A$ define $w^{-1}=x_{n}^{-1} \ldots x_{1}^{-1}$. Let $\Phi$ be a map from $A$ to $B^{*}$, such that $\Phi\left(a_{i}\right)$ is freely reduced and $\Phi\left(a_{i}^{-1}\right)=\Phi\left(a_{i}\right)^{-1}$, for all $i$. Then $\Phi$ induces a map, also denoted $\Phi$, from $A^{*}$ to $B^{*}$. Let $u \in A^{*}$ and $w$ be the word obtained from $\Phi(u)$ by free reduction. Then we say that $(\Phi, u)$ is a representation of $w$. If $\Phi(u)$ is cyclically reduced then $(\Phi, u)$ is said to be a non-cancelling representation of $w$.

Given a Wicks form $[u]$ of genus $g$ we say that $(\Phi, u)$ is a genus $g$ Wicks representation of $w \in B^{*}$ if there is $u^{\prime} \in[u]$ such that $\left(\Phi, u^{\prime}\right)$ is a representation of $w$. If $u$ and $v$ are isomorphic genus $g$ Wicks forms then there exists a genus $g$ Wicks representation $(\Phi, u)$ of $w$ if and only if there exists a genus $g$ Wicks representation $(\Psi, v)$ of $w$. If there exists a genus $g$ Wicks representation of $w$ then there also exists a non-cancelling genus $g$ representation of $w$ (see [3]). 
Definition 2. For $w \in B^{*}$ we define $M(g, w)$ to be the number of isomorphism classes $I$ of maximal Wicks forms over $A$, of genus $g$, such that there exists a noncancelling Wicks representation $(\Phi, u)$ of $w$, for some $u \in I$.

We shall say a word $w \in B^{*}$ has genus $g$ if $w$ has a genus $g$ Wicks representation but no Wicks representation of genus less than $g$. If $w$ does not have a Wicks representation, of any genus, we define its genus to be $\infty$. A cyclically reduced word $w$ has genus $g<\infty$ if and only if it represents an element of the commutator subgroup of the free group on $\left\{b_{1}, \ldots, b_{n}\right\}$ (see [3]).

\section{Words with many representations}

In the sequel, if $u \in A^{*}$ and $w \in B^{*}$, for alphabets $A$ and $B$, and there exists a non-cancelling representation $(\Phi, u)$ of $w$ then we shall say that $w$ is obtained from $u$ by non-cancelling substitution.

Theorem. There is a sequence $w_{2}, w_{3}, w_{4}, \ldots$ of words over an alphabet $B$ of size 24 such that

(i) $w_{g}$ has genus $g$ and

(ii) $M\left(g, w_{g}\right)>g$ !, for $g>10^{10}$.

Proof. Consider a maximal Wicks form $w$ of genus $g$ and length $12 g-6$. We colour vertices of the graph $\Gamma$ of $w$ so that no edge is incident to two vertices of the same colour. A straightforward induction on the number of vertices shows that, for a simple graph whose vertices have degree at most $d$, vertices may be coloured in this way using at most $d+1$ colours. In our case $\Gamma$ has no vertex of degree more than 3 (in fact is regular of degree 3) so four colours 1, 2, 3, 4 are sufficient.

We construct a new labelled oriented graph $\Gamma^{\prime}$ on the surface $S$ associated to $w$, as follows. The underlying graph of $\Gamma^{\prime}$ is the barycentric subdivison of $\Gamma$. Thus $\Gamma^{\prime}$ has two types of vertex; the original vertices of $\Gamma$ which are of degree 3, and new vertices of degree 2 corresponding to edges of $G$. All edges of $G^{\prime}$ join a vertex of degree 3 to a vertex of degree 2 and we orient each edge from the degree 3 vertex to the degree 2 vertex. The vertices of $\Gamma^{\prime}$ of degree 3 inherit a colouring from the corresponding vertices of $\Gamma$. Now let $B=\left\{\alpha_{j}^{ \pm 1}, \beta_{j}^{ \pm 1}, \gamma_{j}^{ \pm 1} \mid j=1,2,3,4\right\}$ be an alphabet disjoint from $A$. Let $u$ be a vertex of $\Gamma^{\prime}$ of degree 3 and colour $j$. Choose one of the 3 edges emanating from $u$ and call it $e_{1}$. Choose one of the other edges and call it $e_{2}$ and call the third $e_{3}$. Label the oriented edges $e_{1}, e_{2}$ and $e_{3}$ coming out of $u$ with $\alpha_{j}, \beta_{j}$ by $\gamma_{j}$, respectively, as shown in Figure 1. Repeat this for all degree 3 vertices of $\Gamma^{\prime}$. Now reading the oriented circuit $C$ with this labelling we obtain a word $v \in B^{*}$. Moreover, the condition on the colouring of vertices of $\Gamma$ means that $v$ is obtained from $w$ by non-cancelling substitution. 


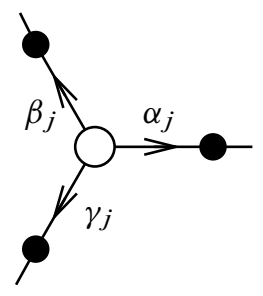

Figure 1. Labelling of edges of $\Gamma^{\prime}$.

To simplify the estimation of the lower bound below we now make a further adjustment to the current labelling of $\Gamma^{\prime}$. Let $u$ be a vertex of degree 3 with outgoing edges labelled $\alpha_{j}, \beta_{j}, \gamma_{j} \in B$. Then either $\alpha_{j}^{-1} \beta_{j}$ or $\beta_{j}^{-1} \alpha_{j}$ is a factor of $w^{\prime \prime}$. Moreover, if $\alpha_{j}^{-1} \beta_{j}$ is a factor of $w^{\prime \prime}$ then so are $\beta_{j}^{-1} \gamma_{j}$ and $\gamma_{j}^{-1} \alpha_{j}$, whereas if $\beta_{j}^{-1} \alpha_{j}$ is a factor then so are $\alpha_{j}^{-1} \gamma_{j}$ and $\gamma_{j}^{-1} \beta_{j}$. In the latter case we alter the labelling on the edges incident to $u$ by interchanging labels $\gamma_{j}$ and $\beta_{j}$ : as shown in Figure 2, where the curved arcs indicate the direction in which the boundary of $S \backslash \Gamma^{\prime}$ is read. The

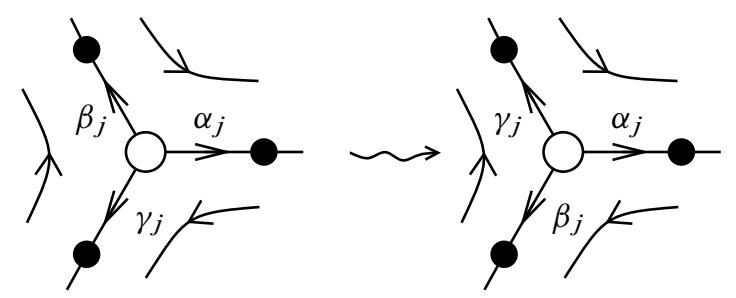

Figure 2. Adjustment of labels on $\Gamma^{\prime}$.

result is that $\alpha_{j}^{-1} \beta_{j}, \beta_{j}^{-1} \gamma_{j}$ and $\gamma_{j}^{-1} \alpha_{j}$ are subwords of the (altered) word $w^{\prime \prime}$. We perform this alteration, if necessary, on every vertex, of degree 3 , of $\Gamma^{\prime}$. By reading the circuit $C$ with these altered labels we obtain a new word $v$; also obtained from $w$ by non-cancelling substitution. Furthermore this word $v \in B^{*}$ satisfies the following conditions.

(i) Every letter of $v$ from $B^{+}$of colour $j$ is followed by a letter of colour $k \neq j$ from $B \backslash B^{+}$.

(ii) Every letter of $v$ from $B \backslash B^{+}$is followed by a letter of the same colour from $B^{+}$.

(iii) None of $\beta_{j}^{-1} \alpha_{j}, \alpha_{j}^{-1} \gamma_{j}$ or $\gamma_{j}^{-1} \beta_{j}$ occur as subwords of $v$.

(iv) The length of $v$ is $24 g-12$.

An example of this construction is given after the proof.

Let us show that $v$ cannot be obtained by a non-cancelling substitution from a Wicks form of genus $k$, where $k<g$. Suppose that $(\Phi, u)$ is a genus $k$ non- 
cancelling representation of $v$. Since a Wicks form of genus $k$ has length at most $12 k-6 \leq 12(g-1)-6$ and $v$ has length $24 g-12$ there must be some letter $a$ occurring in $u$ such that $\Phi(a)>2$. Let $a$ be such a letter. Then $\Phi(a)$ contains a subword $x y z$, where $x, y, z \in B$. As $a^{-1}$ also occurs in $u$ both $x y z$ and $z^{-1} y^{-1} x^{-1}$ must be subwords of $v$. However, from (i) and (ii) above, positive and negative powers of letters of $v$ alternate. Moreover, if $j$ is fixed and $x, y \in\left\{\alpha_{j}, \beta_{j}, \gamma_{j}\right\}$ then $v$ may have subword $x^{-1} y$ but does not have subword $x y^{-1}$. This means that a subword of $v$ of length three always has form $x_{i}^{-1} y_{i} z_{j}^{-1}$ or $x_{i} y_{j}^{-1} z_{j}$, where $x_{i}^{ \pm 1}, y_{i}^{ \pm 1}, y_{j}^{ \pm 1}$ and $z_{j}^{ \pm 1}$ are elements of $B$. Therefore $v$ cannot contain both subwords $x y z$ and $z^{-1} y^{-1} x^{-1}$, and the result follows. Hence the genus of $v$ is $g$.

Now we wish to count the number of words $v$ that may arise in this way. First note that we may read the word $v$ starting from any vertex of $\Gamma^{\prime}$ and so we may choose $v$ so that it begins with $\alpha_{j}$, for some $j \in\{1,2,3,4\}$. Let $V(g)$ denote the set of all freely reduced words of $B^{*}$ which satisfy (i) to (iv) above and which begin with $\alpha_{j}$, for some $j$; so $v \in V(g)$. If $u$ belongs to $V(g)$ and $x \in\left\{\alpha_{s}, \beta_{s}, \gamma_{s}\right\}$ occurs in $u$ then the letter following $x$ belongs to $\bigcup_{i \neq s}\left\{\alpha_{i}^{-1}, \beta_{i}^{-1}, \gamma_{i}^{-1}\right\}$; so there are nine possibilities for this letter. If $y$ is a letter of $B \backslash B^{+}$occurring in $u$ then the letter following $y$ is completely determined by conditions (ii) and (iii) above. Given that there are 4 choices for the first letter $\alpha_{j}$ of $u$ and that $u$ has length $24 g-12$ this means that $|V(g)|=4 \cdot 9^{12 g-7}$.

Let $M(g)=\max \{M(g, v) \mid v$ is a reduced word of genus $g$ and length $24 g-12\}$ and let $W(g)$ be the number of isomorphism classes of maximal oriented Wicks forms of genus $g$. Then $|V(g)| M(g) \geq W(g)$. However it follows from [1] that $W(g)$ grows faster than $g$ !; in fact setting

$$
m(g)=\left(\frac{1}{12}\right)^{g} \frac{(6 g-4) !}{g !(3 g-2)}
$$

we have $W(g) \geq m(g)$. (In [1] $m(g)=m_{1}^{g}$.) Using upper and lower bounds for $n$ !, from [8], straightforward calculations give

$$
M(g) \geq W(g) / M(g) \geq m(g) /\left(4 \cdot 9^{12 g-7}\right)>g !
$$

for $g>10^{10}$, and the statement of the theorem follows.

Example. The quadratic word

$$
w=a_{1} a_{2} a_{3} a_{4} a_{5} a_{1}^{-1} a_{6} a_{2}^{-1} a_{5}^{-1} a_{7} a_{8} a_{3}^{-1} a_{6}^{-1} a_{9} a_{7}^{-1} a_{4}^{-1} a_{8}^{-1} a_{9}^{-1}
$$

gives rise to the labelled graph of Figure 3, embedded on an orientable surface of genus 2; so this word is a maximal Wicks form of genus 2. (The small curved arrows indicate the direction to be taken at each vertex in reading the circuit $C$.) A colouring of the graph with colours 1, 2 and 3 is also shown in the figure.

The barycentric subdivision of this graph with its new orientation and a choice of new labelling is shown in Figure 4. 


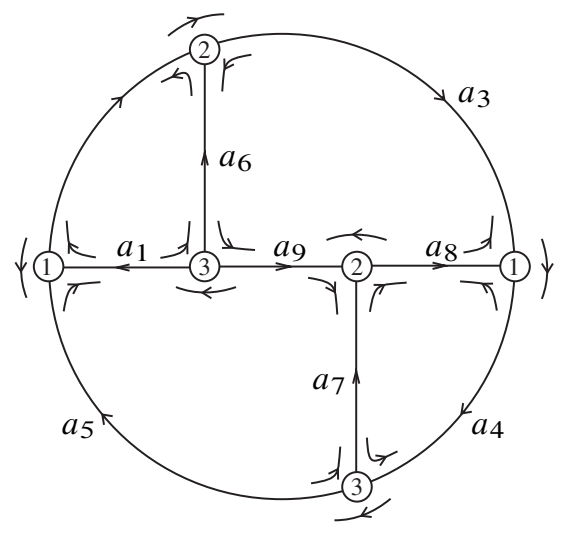

Figure 3. A maximal Wicks form.

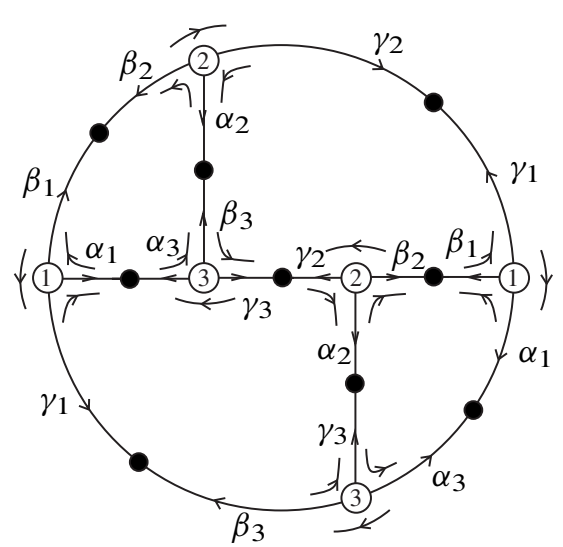

Figure 4. Barycentric subdivision and new labelling.

Starting by reading the edge labelled $\alpha_{1}$ coming out of the rightmost vertex we obtain the word

$$
\begin{gathered}
v=\alpha_{1} \alpha_{3}^{-1} \beta_{3} \gamma_{1}^{-1} \alpha_{1} \alpha_{3}^{-1} \beta_{3} \alpha_{2}^{-1} \beta_{2} \beta_{1}^{-1} \gamma_{1} \beta_{3}^{-1} \gamma_{3} \alpha_{2}^{-1} \beta_{2} \beta_{1}^{-1} \gamma_{1} \gamma_{2}^{-1} \\
\alpha_{2} \beta_{3}^{-1} \gamma_{3} \gamma_{2}^{-1} \alpha_{2} \gamma_{3}^{-1} \alpha_{3} \alpha_{1}^{-1} \beta_{1} \beta_{2}^{-1} \gamma_{2} \gamma_{3}^{-1} \alpha_{3} \alpha_{1}^{-1} \beta_{1} \beta_{2}^{-1} \gamma_{2} \gamma_{1}^{-1} .
\end{gathered}
$$

No adjustment of the labels is required as $v$ contains none of the forbidden subwords.

As mentioned in the introduction the objects counted in [2] are equivalence classes of representations of particular words $w_{g}$; whereas here we count (isomorphism classes of) representations themselves. It seems plausible that by choosing the words $w_{g}$ carefully the size of equivalence classes could be made small, but we have no 
proof of any such claim. We can however offer some evidence that it is possible, by making a moderate increase in the size of the alphabet, to choose the words $w_{g}$ from a much more limited set than $V(g)$ above. A word in $B^{*}$ is said to be square-free if it contains no subword of the form $u u$, where $u$ is a non-trivial element of $B^{*}$. Such square-free words exist (see for example [7], Chapter 4).

Corollary. In the theorem above, replacing $B$ with an alphabet of size 80, the words $w_{g}$ may be chosen to be square-free and the conclusion of the theorem then holds for $g>10^{17}$.

Proof. Let $w$ of be a maximal Wicks form of genus $g$ and length $12 g-6$ and, as in the proof of the theorem, let $v$ be the label of the oriented circuit $C$ on the surface corresponding to $w$ with the labelling constructed in the proof. Now for each letter $x_{j} \in\left\{\alpha_{j}, \beta_{j}, \gamma_{j}\right\} \subseteq B$, where $j=1,2,3$ or 4 , define a set $E^{+}\left(x_{j}\right)=$ $\left\{x_{j, 1}, x_{j, 2}, x_{j, 3}\right\}$ and define $E\left(x_{j}\right)=E^{+}\left(x_{j}\right) \cup\left\{x^{-1} \mid x \in E^{+}\left(x_{j}\right)\right\}$ and $E=$ $\bigcup_{j=1}^{4}\left(E\left(\alpha_{j}\right) \cup E\left(\beta_{j}\right) \cup E\left(\gamma_{j}\right)\right)$. Further let $E_{S}=\left\{x_{1,0}^{ \pm 1}, x_{2,0}^{ \pm 1}, x_{3,0}^{ \pm 1}, x_{4,0}^{ \pm 1}\right\}$ and let $\hat{E}=E \cup E_{S}$.

Fix some element $x_{i}^{+1} \in B$ which occurs in $v$. If $x_{i}$ occurs $s$ times in $v$, then $v$ can be written as $v=u_{1} x_{i} u_{2} x_{i} u_{3} \ldots x_{i} u_{s} x_{i} u_{s+1}$ where the words $u_{1}, u_{2}, \ldots, u_{s}, u_{s+1}$ do not contain $x_{i}$ (they contain $x_{i}^{-1}$, of course, but we are interested in positive powers of $x_{i}$ only). Now $v$ is transformed as follows. Choose a square-free word $t_{1} \ldots t_{s-1}$ of length $s-1$ in $E\left(x_{i}\right)^{*}$. Replace the first occurrence of $x_{i}$ with $x_{i, 0}$ and replace the $j$ th occurrence of $x_{i}$ with $t_{j-1}$, for $j=2, \ldots, s$. Reading the oriented circuit $C$ each occurrence of $x_{i}$ is paired to an occurrence of $x_{i}^{-1}$ in $v$; as each edge is read in both directions. The occurrence $x_{i}^{-1}$ paired to the $j$ th occurrence of $x_{i}$ is now replaced by $x_{i, 0}^{-1}$ if $j=1$, and by $t_{j-1}^{-1}$ otherwise. (The special letter $x_{i, 0}$ is introduced to ensure that all cyclic permutations of the resulting word are square-free, as we are dealing with cyclic words.) Repeat this transformation with respect to every letter of positive exponent occurring in $v$ and denote the resulting word by $z$. Then $z \in \hat{E}^{*}$ is obtained by non-cancelling substitution from $w$.

It is easy to see that $z$ is square-free as follows. Suppose not: then $z$ has a subword $u u$. Let $u=r_{1} r_{2} \ldots r_{p}$, where $r_{i} \in E$. Set $j_{1}=1$ if $r_{1}$ is an element of $E$ of exponent +1 and set $j_{1}=2$ otherwise. Since $z$ consists of alternating positive and negative powers then $r_{j_{1}}$ will be a positive power. Then $r_{j_{1}}=e_{i} \in E\left(x_{i}\right)$, for some $i$. Suppose that there are $p$ occurrences of $e_{i}$ in $u$, namely $r_{j_{1}}, \ldots, r_{j_{p}}$, with $j_{1}<\cdots<j_{p}$. Since $u u$ is a subword of $z$ the word $r_{j_{1}} \ldots r_{j_{p}} r_{j_{1}} \ldots r_{j_{p}}$ is a subword of the word $t=t_{1} \ldots t_{s-1}$ used to construct $z$, above. This contradicts the choice of $t$ as a square-free word. Hence $z$ is square-free as claimed.

The argument of the proof of the theorem shows that $z$ has genus $g$. As $v$ begins with $\alpha_{j}$ the first letter of $z$ is $x_{j, 0}$ and so there are 4 possibilities for this letter. Each other letter of $z$ of positive exponent is obtained by substitution of one of the three elements of $E\left(x_{j}\right)$ for $x_{j}$, for some $x_{j} \in B^{+}$; or is the first letter of its kind in $z$ in 
which case it is a uniquely determined element of $E_{S}$. It follows that there are at most $4 \cdot 27^{12 g-7}$ possibilities for $z$. The result now follows.

Acknowledgements. The authors are indebted to A. G. Miasnikov for his useful comments and suggestions on this work.

\section{References}

[1] R. Bacher and A. Vdovina, Counting 1-vertex triangulations of oriented surfaces. Discrete Math. 246 (2002), 13-27. Zbl 0994.05058 MR 1884884

[2] M. Bestvina and M. Feighn, Counting maps from a surface to a graph. Geom. Funct. Anal. 15 (2005), 939-961. Zbl 05045833 MR 2221155

[3] L. P. Comerford, Jr. and C. C. Edmunds, Products of commutators and products of squares in a free group. Internat. J. Algebra Comput. 4 (1994), 469-480. Zbl 0808.20027 MR 1297152

[4] M. Culler, Using surfaces to solve equations in free groups. Topology 20 (1981), 133-145. Zbl 0452.20038 MR 605653

[5] M. Dehn, Papers on group theory and topology. Springer-Verlag, New York 1987. MR 0881797

[6] L.Mosher, A user's guide to the mapping class group: once punctured surfaces. In Geometric and computational perspectives on infinite groups, DIMACS Ser. Discrete Math. Theoret. Comput. Sci. 25, Amer. Math. Soc., Providence, RI, 1996, 101-174. Zbl 0851.57015 MR 1364183

[7] M. Lothaire, Algebraic combinatorics on words. Encyclopedia Math. Appl. 90, Cambridge University Press, Cambridge 2002. Zbl 1001.68093 MR 1905123

[8] H. Robbins, A remark on Stirling's formula. Amer. Math. Monthly 62 (1955), 26-29. Zbl 0068.05404 MR 0069328

[9] A. A. Vdovina, Constructing of orientable Wicks forms and estimation of their number. Comm. Algebra 23 (1995), 3205-3222. Zbl 0836.20043 MR 1335298

Received August 14, 2008; revised September 15, 2008

A. Duncan, School of Mathematics and Statistics, Newcastle University, Newcastle upon Tyne, NE1 7RU, United Kingdom

E-mail: a.duncan@ncl.ac.uk

A. Vdovina, School of Mathematics and Statistics, Newcastle University, Newcastle upon Tyne, NE1 7RU, United Kingdom

E-mail: Alina.Vdovina@newcastle.ac.uk 